Artigo Original

\title{
Qualidade de vida dos trabalhadores de enfermagem de unidades de terapia intensiva*
}

\author{
Quality of life among nursing assistants and licensed practical nurses from intensive care units
}

Calidad de vida de trabajadores de enfermería de unidades de cuidados intensivos

Simone Paschoa ${ }^{1}$, Suely Sueko Viski Zanei ${ }^{2}$, Iveth Yamaguchi Whitaker ${ }^{3}$

\begin{abstract}
RESUMO
Objetivos: Avaliar a qualidade de vida (QV) dos técnicos e auxiliares de enfermagem que trabalham em Unidades de Terapia Intensiva e identificar os fatores sociodemográficos e relacionados ao trabalho que podem influenciar a QV. Método: Estudo transversal realizado em um hospital escola da cidade de São Paulo. A coleta de dados de 126 trabalhadores de enfermagem em nove Unidades de Terapia Intensiva foi realizada em outubro e novembro de 2005. Utilizou-se como instrumento de avaliação de QV o WHOQOL-BREF. Resultados: As médias em cada domínio da QV foram: Relações Sociais 66,3; Psicológico 60,8; Físico 53,1 e Meio-Ambiente 49,4. A variável idade apresentou correlação positiva somente com o domínio físico e o número de empregos se correlacionou inversamente com os domínios Físico, Psicológico e Relações Sociais. Conclusão: A qualidade de vida dos trabalhadores de enfermagem em todas as dimensões é relativamente baixa, considerando-se os valores entre 0-100. Há fraca correlação dos domínios com idade e número de empregos.
\end{abstract}

Descritores: Qualidade de vida; Unidades de terapia intensiva; Equipe de enfermagem; Questionário

\begin{abstract}
Objectives: To assess the quality of life of nursing assistants and licensed practical nurses from nine Intensive Care Units and to determine whether their demographic characteristics influence their quality of life. Methods: This cross-sectional study was conducted in a teaching hospital in São Paulo city, Brazil. The sample consisted of 126 nursing assistants or licensed practical nurses. Data were collected from October to November, 2005. Quality of life was measured with the WHOQOL-BREF tool. Results: The average score on each domain of the WHOQOL-BREF were: Social relations (66.3), psychological (60.8), physical (53.1), and environment (49.4). Among the demographics, age was positively correlated with the physical domain of quality of life, and number of jobs was inversely correlated with the physical, psychological, and social relations domains. There was also a weak correlation between age and number of jobs. Conclusion: The scores on the domains of quality of life among the sample of this study were low.
\end{abstract}

Keywords: Quality of life; Intensive care units; Nursing team; Questionnaries

\section{RESUMEN}

Objetivos: Evaluar la calidad de vida (CV) de los técnicos y auxiliares de enfermería que trabajan en Unidades de Cuidados Intensivos e identificar los factores sociodemográficos y los relacionados al trabajo que pueden influir en la CV. Métodos: Se trata de un estudio transversal realizado en un hospital escuela de la ciudad de Sao Paulo. La recolección de datos de 126 trabajadores de enfermería en nueve Unidades de Cuidados Intensivos se llevó a cabo en octubre y noviembre del 2005. Se utilizó como instrumento de evaluación de CA el WHOQOL-BREF. Resultados: Los promedios en cada dominio de la CV fueron: Relaciones Sociales 66,3; Psicológico 60,8; Físico 53,1 y Medio-Ambiente 49,4. La variable edad presentó correlación positiva solamente con el dominio físico y el número de empleos se correlacionó inversamente con los dominios Físico, Psicológico y Relaciones Sociales. Conclusión: La calidad de vida de los trabajadores de enfermería en todas las dimensiones es relativamente baja, considerándose los valores entre 0-100. Hay una débil correlación de los dominios con la edad y número de empleos.

Descriptores: Calidad de vida; Unidades de Cuidados Intensivos; Grupo de enfermería; Cuestionario

\footnotetext{
* Trabalho realizado no Hospital São Paulo da Universidade Federal de São Paulo - UNIFESP.

1 Acadêmica do Curso de Especialização em Enfermagem em UTI-Adulto - Modalidade Residência da Universidade Federal de São Paulo - UNIFESP-São Paulo (SP), Brasil.

${ }^{2}$ Doutora em Enfermagem pelo Departamento de Enfermagem da Universidade Federal de São Paulo - UNIFESP-São Paulo (SP), Brasil.

${ }^{3}$ Doutora em Enfermagem do Departamento de Enfermagem da Universidade Federal de São Paulo - UNIFESP- São Paulo (SP), Brasil.
} 


\section{INTRODUÇÃO}

A população de pacientes nas Unidades de Terapia Intensiva (UTIs) é assistida por uma equipe multiprofissional e interdisciplinar, na qual os profissionais de enfermagem são numericamente superiores a outros profissionais na maioria das instituições de saúde.

Historicamente, a equipe de enfermagem é composta, em sua maioria, por mulheres. Considerando-se o contexto socioeconômico atual, pode-se inferir que grande parte do contingente dessas trabalhadoras estão sujeitas a vivenciar conflitos em razão das exigências profissionais e de sua vida pessoal, em razão da dupla ou tripla jornada de trabalho. Além disso, há que se considerar que o hospital é uma instituição que utiliza escalas de turnos pela necessidade da manutenção das atividades durante 24 horas ininterruptas, mesmo nos finais de semana ou feriados. Assim sendo, ressalta-se o prejuízo dessas trabalhadoras na participação de atividades como as escolares, culturais, sociais, entre outras, além de estarem se submetendo a uma carga mental excessiva de trabalho ${ }^{(1-3)}$.

Um dos fatores de desgaste físico e psicológico para os trabalhadores da área da saúde é o acúmulo de dois ou mais vínculos empregatícios. No ambiente das UTIs, a proximidade com os pacientes é intensa, visto que todos são altamente dependentes. Além disso, muitos deles em alguma fase da doença tornam-se extremamente "pesados" ou "trabalhosos" em razão da gravidade ou da condição física. A presença, por exemplo, do edema generalizado, curativos extensos ou utilização de vários artefatos dificultam as manipulações e aumentam o consumo do tempo para a execução dos cuidados. Tais exigências somadas ao duplo vínculo podem levar muitos trabalhadores de enfermagem ao processo de desgaste físico, advindo da sobrecarga de trabalho.

As UTIs, em geral, possuem uma rotina permeada de incertezas, instabilidade, imediatismo e variabilidade, podendo ser geradoras de estresse aos profissionais da equipe multidisciplinar. Além disso, particularmente para os profissionais de enfermagem, as condições de trabalho podem ser insatisfatórias em decorrência de inúmeros fatores: baixa remuneração, hierarquização, diversidade e complexidade dos procedimentos técnicos ${ }^{(4)}$.

Assim, considera-se importante avaliar a qualidade de vida dos trabalhadores de enfermagem, uma vez que a mesma está diretamente relacionada a diversos aspectos da vida cotidiana, dentre elas, as condições de trabalho.

A Qualidade de vida (QV), segundo a Organização Mundial da Saúde - OMS, é "a percepscão do indivíduo de sua posição na vida no contexto da cultura e sistema de valores nos quais ele vive e em relação aos seus objetivos, expectativas, padrões e preocupacões" ${ }^{\prime(5)}$. É considerado um conceito abstrato, subjetivo e multidimensional por envolver vários aspectos da vida humana tais como: relações sociais, saúde, família, trabalho, meio-ambiente, dentre outros. É dinâmico e pode ser influenciado por aspectos culturais, religiosos, éticos e valores pessoais ${ }^{(5)}$.

Indiretamente, a má QV em uma ou mais dimensões (por exemplo, física e/ou emocional) dos profissionais da equipe de saúde pode comprometer a dinâmica de atendimento, gerando prestação inadequada de serviços, com prejuízo institucional e, principalmente, para a assistência aos pacientes.

Assim sendo, considerando-se que o trabalho é um dos fatores que pode influenciar a qualidade de vida das pessoas, os objetivos do presente estudo foram:

* Avaliar a qualidade de vida dos técnicos (TE) e auxiliares de enfermagem (AE) atuantes em Unidades de Terapia Intensiva de um hospital escola.

* Identificar os fatores sociodemográficos e relacionados ao trabalho que podem influenciar a $\mathrm{QV}$ desses trabalhadores.

\section{MÉTODOS}

Trata-se de um estudo transversal realizado em hospital escola, terciário, de grande porte localizado na cidade de São Paulo. Após a aprovação do estudo pelo Comitê de Ética da Instituição e obtenção da autorização das chefias de enfermagem de nove Unidades de Terapia Intensiva, nos meses de outubro e novembro de 2005 realizou-se a coleta de dados. A amostra foi composta por $126 \mathrm{TE}$ e AE das unidades citadas, que concordaram em participar da pesquisa e assinaram o Termo de Consentimento Livre e Esclarecido.

Cada participante recebeu dois questionários autoaplicáveis. O primeiro, contendo questões sobre o perfil sociodemográfico e condições de trabalho e o segundo, o instrumento específico de avaliação de QV, WHOQOL-BREF ${ }^{(5-6)}$.

O WHOQOL-BREF é um instrumento de avaliação da qualidade de vida, elaborado em 1998 por um grupo multicentrico interessado em estudos sobre QV (WHOQOL Group), da OMS ${ }^{(5-7)}$. Este instrumento é derivado do WHOQOL-100, assim denominado por conter 100 questões que agrupadas formam seis dimensões ou domínios: físico, psicológico, nível de independência, relações sociais, ambiente e espiritualidade/religiosidade/crenças pessoais. A versão abreviada, WHOQOL-BREF, é composta por 26 questões baseadas em quatro domínios: físico, psicológico, relações sociais e meio-ambiente. O escore para cada domínio pode ser transformado em uma escala que varia de $0-100$, sendo zero o pior e 100 o melhor resultado. Estes instrumentos foram idealizados dentro de uma perspectiva internacional, com o objetivo de abranger as diversas culturas mundiais, uma vez que tornaram-se crescentes os estudos sobre qualidade de 
vida, a partir da década $90^{(7-8)}$.

As análises estatísticas foram realizadas no programa SPSS -13 (Statistical Package for the Social Sciences - versão 13). As questões relacionadas ao WHOQOL-BREF foram recodificadas seguindo-se os passos recomendados para pontuação dos escores ${ }^{(6-8)}$. A verificação da confiabilidade do instrumento para essa população foi verificada através do Coeficiente Alfa de Cronbach. Para as análises descritivas dos dados sociodemográficos e condições de trabalho, foram utilizadas medidas de tendência central e medidas de dispersão.

Para verificar a relação entre as variáveis estudadas e os domínios do WHOQOL-BREF, foram aplicados os seguintes testes: Kolmogorov-Smirnov para verificar se as variáveis quantitativas contínuas apresentavam distribuição normal, teste de Kruskal - Wallis e a Análise de Variância (ANOVA-Oneway) para análise de dados contínuos que não apresentaram distribuição normal e possuíam três ou mais categorias. Para a análise das variáveis qualitativas categóricas foi utilizado o teste Qui - Quadrado e para as variáveis quantitativas foi utilizado o Coeficiente de Correlação de Spearman.

Especificamente, em relação à classificação da magnitude das correlações entre as variáveis foram adotados os valores recomendados pela BMJ - British Medical Journal: 0,00-0,19 ausente ou muito fraca; 0,20 - 0,39 fraca; 0,40 - 0,59 moderada; 0,60 - 0,79 forte e $0,80-1,00$ muito forte ${ }^{(9)}$.

Neste estudo, os resultados foram considerados estatisticamente significativos quando o nível descritivo foi menor que $5 \%(\mathrm{p}<0,05)$.

\section{RESULTADOS}

A população de TE e AE nas UTIs, segundo as escalas setoriais, totalizou 198 trabalhadores. Entretanto, ao longo do período, foi deflagrado estado de greve na instituição, o que ocasionou ausências. Assim, a amostra foi constituída de 126 trabalhadores da equipe de enfermagem, 63,6\% do total.

Quanto às características sociodemográficas e profissionais, 104 eram do sexo feminino (85,5\%). A faixa etária média foi de 30,9 anos (mínimo 20,0; máximo 56,0 e DP $\pm 8,5)$, sendo que $63(50 \%)$ eram solteiras e 45 $(35,7 \%)$ eram casadas. Quanto à escolaridade, 88,9\% possuíam o nível técnico de ensino e 51,6\% relataram estudar e trabalhar concomitantemente.

Em relação ao número de empregos, 85 (67,5\%, DP \pm $0,5)$ das entrevistadas dedicavam-se profissionalmente a apenas um emprego. Entre as que possuíam dois ou mais vínculos empregatícios verificou-se que o segundo emprego era predominantemente na área da saúde (90,2\%), com uma média de 47,8 horas (máximo 84, mínimo 30 e DP \pm 15,1) de trabalho semanal.

Os turnos de trabalho foram variados, sendo estes: $44(34,9 \%)$ à noite, $35(27,8 \%)$ pela manhã, $24(19,0 \%)$ a tarde. Dentre as que possuíam dois empregos, $9(7,1 \%)$ trabalhavam a tarde e a noite, 7 (5,6\%) durante a manhã e a tarde, $4(3,2 \%)$ pela manhã e a noite. Três participantes $(2,4 \%)$ referiram possuir três vínculos e trabalhavam nos três períodos. Considerando-se o segundo emprego, os setores de trabalho foram: UTI-Adulto (45,5\%), UTIPediátrica (18,2\%), Enfermaria $(21,2 \%)$ e outros setores $(15,1 \%)$. Em relação à renda, $3(2,4 \%)$ participantes não informaram; $56(44,4 \%)$ relataram possuir uma renda pessoal mensal de 2 a 4 salários mínimos, 47 (37,3\%) recebiam de 5 a 6 salários e $20(15,9 \%)$ acima de 6 salários mínimos.

Quanto à avaliação da QV das entrevistadas, dois questionários foram devolvidos em branco, totalizando 124 participantes. O instrumento utilizado - WHOQOLBREF - foi avaliado quanto a sua confiabilidade através da análise de consistência interna dos itens, utilizando-se o Coeficiente Alfa de Cronbach. O resultado obtido foi de 0,915 , atestando que o instrumento apresentou excelente confiabilidade para a população estudada. Quanto aos domínios da QV, a Tabela 1 mostra os resultados encontrados.

A Tabela 1 possibilita constatar o domínio relações sociais, como sendo o melhor aspecto da QV dos TE e AE. Em segundo lugar, tem-se o domínio psicológico, o físico como terceiro, e por último, o domínio meioambiente.

Procurando responder ao segundo objetivo deste estudo, foram levantados os itens relacionados às condições sociodemográficas e profissionais, que

Tabela 1 - Média dos escores da avaliação de qualidade de vida dos técnicos e auxiliares de enfermagem de UTIs segundo domínios do WHOQOL- BREF. São Paulo, 2005.

\begin{tabular}{lrrrrrr}
\hline Domínios do WHOQOL-BREF & $\mathbf{N}$ & Mínimo & Máximo & Média & Desvio Padrão \\
\hline Físico & 124 & 25,0 & 85,7 & 53,1 & $\pm 11,9$ \\
Psicológico & 124 & 29,1 & 87,5 & 60,8 & $\pm 11,9$ \\
Relações Sociais & 124 & 8,3 & 100,0 & 66,3 & $\pm 18,0$ \\
Meio-Ambiente & 124 & 18,7 & 90,6 & 49,4 & $\pm 14,5$ \\
\hline
\end{tabular}


poderiam correlacionar-se com os domínios da QV. As variáveis que apresentaram alguma correlação foram a idade e o número de empregos, conforme apresentado na Tabela 2. As variáveis escolaridade, renda pessoal e turno de trabalho não evidenciaram correlações com os domínios de QV segundo o WHOQOL-BREF.

Tabela 2 - Correlação de Spearman entre os escores dos domínios do WHOQOL-BREF com variáveis sociodemográficas e profissionais. São Paulo, 2005.

\begin{tabular}{lcc}
\hline $\begin{array}{c}\text { Domínios do } \\
\text { WHOQOL- } \\
\text { BREF }\end{array}$ & \multicolumn{2}{c}{ Variáveis } \\
\cline { 2 - 3 } & \multicolumn{1}{c}{ Idade } & $\mathbf{N}^{\mathbf{o}}$ de Empregos \\
\hline Físico & $0,229 /(0,012)^{*}$ & $-0,222 /(0,013)^{*}$ \\
Psicológico & $0,153 /(0,094)$ & $-0,209 /(0,020)^{*}$ \\
Relações Sociais & $0,067 /(0,467)$ & $-0,277 /(0,002)^{*}$ \\
Meio-ambiente & $0,119 /(0,194)$ & $-0,089 /(0,324)$ \\
\hline Correlação significativa $\mathrm{p}<0,05$. &
\end{tabular}

$\mathrm{Na}$ Tabela 2, a correlação positiva e significativa da idade pode ser observada somente com o domínio físico. A correlação positiva, nesse caso, significa que quanto maior a idade, maior o escore nesse domínio. A magnitude da correlação foi, entretanto, de fraca intensidade. Quanto ao número de empregos, há correlação negativa ou inversa, apontando que quanto maior o número de empregos, menor o escore dos domínios correlacionados. Igualmente, essas correlações foram de fraca magnitude em todos os domínios, sendo significativa nos aspectos físico, psicológico e relações sociais. Para as variáveis: escolaridade, renda pessoal e turnos de trabalho foram utilizados o teste estatístico QuiQuadrado, não sendo encontrado níveis de significância estatística $(p>0,05)$ entre estas e os domínios da QV.

\section{DISCUSSÃO}

A QV geral dos trabalhadores de enfermagem tem sido avaliada em alguns estudos sob diferentes perspectivas, tais como: qualitativa ${ }^{(10)}$, no enfoque $\mathrm{da}$ determinação social ${ }^{(11)}$ ou relacionando-a com as condições de trabalhos em turnos ${ }^{(3,12)}$, principalmente $\mathrm{O}$ noturno $^{(13)}$ ou ainda, relacionando às condições de vida, trabalho e transtornos mentais ${ }^{(14)}$. Outros estudos abordam a qualidade de vida no trabalho(15-17), enfatizando especificamente esse cenário, considerado importante para a satisfação e bem-estar do trabalhador. Os sujeitos dessas diversas pesquisas são os trabalhadores de nível médio (AE e TE), apenas os enfermeiros ${ }^{(10,18)}$, ou ainda, a equipe como um todo ${ }^{(16-17)}$.

Alguns estudos enfocam categorias específicas de enfermagem, tais como enfermeiros-residentes ${ }^{(19)} \mathrm{e}$ enfermeiros-docentes ${ }^{(20)}$. Apesar dos diferentes enfoques, de um modo geral observa-se que a $\mathrm{QV}$ geral dos profissionais de enfermagem tem sido pouco avaliada por meio de instrumentos próprios. Entre os estudos que utilizaram um instrumento de $\mathrm{QV}$, traduzido e validado, dois aplicaram o Medical Outcomes Study 36item Short Form Health Survey (SF-36) entre os trabalhadores de enfermagem de Centro Cirúrgico ${ }^{(21)}$ e entre os residentes de enfermagem ${ }^{(19)}$. Outros instrumentos de QV utilizados para populações gerais são os do grupo WHOQOL (WHOQOL-100 e WHOQOL-BREF) ${ }^{(6,7)}$, porém a aplicação de uma das versões entre trabalhadores de qualquer área é rara, sendo localizado em nosso meio apenas um estudo com médicos- residentes ${ }^{(22)}$.

Nesse contexto, o presente estudo apresenta dados até então desconhecidos e, portanto, de difícil comparação. O que se evidencia é que a QV dos trabalhadores de enfermagem das UTIs, avaliada pelo WHOQOL- BREF, é em todas as dimensões, (física, psicológica, relações sociais e meio-ambiente) relativamente baixa, considerando-se os valores mínimo (pior) e máximo (melhor) entre 0 -100, respectivamente.

O domínio relações sociais apresentou a média mais elevada $(66,3)$. Vale ressaltar, que nesse aspecto, questionase o nível de satisfação com as pessoas do círculo social, o apoio que recebe e a satisfação com a atividade sexual (questões 20, 21 e 22). Considerando-se que, a população estudada foi constituída de trabalhadores, esperava-se que os escores fossem superiores aos observados em populações com algum problema de saúde. De fato, o escore verificado $(66,3)$ nesta amostra foi superior, se comparado, por exemplo, ao de indivíduos idosos com depressão ${ }^{(23)}$ que obtiveram a média de 56,68 e pessoas com dor lombar crônica, cuja média nesse domínio foi de $53,2^{(24)}$. O escore final do domínio na amostra estudada, possivelmente não foi mais elevado, pois cerca de $30,0 \%$ dos respondentes optaram pela alternativa neutra (nem satisfeito/nem insatisfeito) ao responderem as questões.

No domínio psicológico que avalia, por exemplo, se o entrevistado está satisfeito consigo mesmo e com sua aparência ou a freqüência de sentimentos negativos, a média da amostra estudada foi de 60,8. Este valor é superior aos dos idosos depressivos $(49,8)^{(23)}$ ou pacientes esquizofrênicos $(55,8)^{(25)}$, entretanto, não pode ser considerada satisfatória. As questões que possivelmente influenciaram esse escore foram "o quanto aproveita a vida?" (questão 05), e "com que freqüência tem sentimentos negativos?” (questão 26). As respostas muito 
pouco e mais ou menos para a questão 5 totalizaram 79 $(62,7 \%)$ e na questão 26,75 respondentes $(59,5 \%)$ escolheram a alternativa algumas vezes. Percebe-se, portanto, que uma parcela importante da amostra considera não estar aproveitando a vida e que a presença de sentimentos negativos não é rara, o que justificaria o escore relativamente baixo do aspecto psicológico.

Em relação ao domínio físico, os itens principais enfocam a presença de dor ou desconforto, dependência de medicação, satisfação com o sono, capacidade para o trabalho e atividades diárias, entre outros. A amostra de trabalhadores estudada obteve a média de 53,1. Este valor é comparável no mesmo domínio, com os escores de pacientes portadores de $\operatorname{HIV}(53,6)^{(26)}$, pacientes esquizofrênicos $(54,0)^{(25)}$ e pacientes com dor lombar crônica $(52,4)^{(24)}$. Tal resultado indica que a amostra estudada tem algum grau de comprometimento nesse domínio. Ressalta-se que o item referente à qualidade do sono pode estar influenciando no valor da média, uma vez que um terço da amostra trabalha no período noturno e alguns em até dois períodos consecutivos, sendo um deles o noturno. A comprovação da insatisfação com o sono, (questão 16), é vista pela resposta de 45 pessoas $(36,3 \%)$ que optaram por muito insatisfeito ou insatisfeito e $27(21,4 \%)$ escolheram mais ou menos. Essa condição pode deixar o indivíduo sonolento de forma persistente podendo afetar a capacidade para o trabalho, o nível de energia e a capacidade de realizar, satisfatoriamente, as atividades cotidianas ${ }^{(27)}$.

Uma possibilidade para explicar o baixo escore no domínio físico seria a presença de alguma doença, porém esse dado não foi coletado. Todavia, estudos relatam que trabalhadores noturnos possuem um desequilibrio entre os horários das refeições, bem como a mudança da qualidade dos alimentos (alimentos congelados, précozidos), aumento na ingesta de bebidas cafeinadas, dificuldades de digestão, azia, constipação, propensão a problemas cardiovasculares como hipertensão arterial, doenças isquêmicas, maiores riscos de infarto, angina pectoris, entre outros ${ }^{(27)}$. No presente estudo, 29 (23,4\%) trabalhadores informaram estar muito insatisfeitos ou insatisfeitos com a saúde (questão 2); 57 (45,3\%) indivíduos assinalaram que a dor/desconforto os impediu mais ou menos até extremamente, de realizar alguma coisa (questão 3) e $38(30,1 \%)$ informaram necessitar de medicação ou tratamento médico. Esses dados justificam o escore baixo no domínio físico.

O domínio com menor escore foi o meio-ambiente, com valor de 49,4. Nesse domínio estão incluídas perguntas relacionadas à segurança, condições do ambiente físico, dinheiro para as necessidades, lazer, moradia, transporte e acesso aos serviços de saúde. Dentre essas, as poucas oportunidades de lazer (questão 14) afetam 57 indivíduos $(45,2 \%)$ ou aqueles que informam moderadas oportunidades correspondem a 44 pessoas $(34,9 \%)$. A somatória desses dois grupos corresponde a $80,0 \%$ do total, indicando que esse aspecto está longe do desejável para a maioria dos participantes. O outro item que contribuiu para o escore baixo relaciona-se com a quantidade de dinheiro para a satisfação das necessidades (questão 12). As opções nada e muito pouco somam 44 pessoas $(35,0 \%)$ e a opção média quantidade foi escolhida por 63 pessoas $(50,0 \%)$. Outra possível justificativa para o escore baixo pode estar relacionada ao fato de 103 pessoas $(83,7 \%)$ receberem no máximo seis salários mínimos, que como visto, não atende satisfatoriamente a necessidade da maioria dos entrevistados.

Finalizando, vale lembrar que as UTIs são locais de trabalho consideradas de alto risco ocupacional (riscos biológicos, físicos, químicos, ergonômicos e riscos de acidente de trabalho) ${ }^{(28)}$ e essas condições podem ter influenciado a resposta da questão 9 quão saudável é o ambiente físico. As respostas nada e muito pouco totalizaram 45,6\%. Entretanto, o item não é específico e os respondentes poderiam estar se referindo tanto ao ambiente de trabalho como ao ambiente em que vivem.

As análises estatísticas sobre a influência das variáveis sociodemográficas e condições de trabalho revelaram que a idade tem correlação positiva $(0,229)$ com o domínio físico. Já, o número de empregos correlacionou-se negativamente $(-0,222)$ com o domínio físico; com o psicológico $(-0,209)$ e com relações sociais $(-0,277)$. Estas correlações foram significativas $(\mathrm{p}<0,05)$, porém de fraca magnitude, indicando que as variáveis estão correlacionadas, porém, na amostra estudada, não foi possível comprovar que tais correlações são incontestáveis.

\section{CONCLUSÃO}

A qualidade de vida dos AE e TE que trabalham nas UTIs avaliada pelo instrumento WHOQOL-BREF apresentou as seguintes médias: domínio físico $=53,1$; domínio psicológico $=60,8$; relações sociais $=66,3 \mathrm{e}$ meio-ambiente $=49,4$. A variável idade se correlacionou com o domínio físico e número de empregos se correlacionou inversamente com os domínios físico, psicológico e relações sociais.

Esses resultados evidenciaram que os trabalhadores de enfermagem de nível médio que atuam nas UTIs não obtiveram escores satisfatórios nos domínios de QV avaliados pelo WHOQOL-BREF, principalmente nos domínios físico e meio ambiente.

Evidentemente, a instituição ou o local de trabalho, não pode ser responsabilizada globalmente por esses resultados, visto que a $\mathrm{QV}$ envolve outros aspectos da vida além do trabalho. Entretanto, o conhecimento dos aspectos que estão comprometidos pode ser interessante para os gestores de recursos humanos para que possam 
implementar programas voltados ao atendimento de seu pessoal direcionados para as dimensões mais prejudicadas. A melhoria da QV dos trabalhadores de enfermagem pode favorecer a instituição na medida que indivíduos satisfeitos podem melhorar sua produtividade e qualidade do ponto de vista profissional, revertendo, portanto, em melhoria da qualidade da assistência prestada.

\section{REFERÊNCIAS}

1. Spindola T, Santos RS. Mulher e trabalho: a história de vida de mães trabalhadoras de enfermagem. Rev Latinoam Enfermagem. 2003;11(5):593-600.

2. Lopes MJM. O trabalho da enfermeira: nem público, nem privado, feminino, doméstico e desvalorizado. Rev Bras Enfermagem. 1988; 41(3/4):211-7.

3. Marziale MHP, Rozestraten RJA. Turnos alternantes: fadiga mental de enfermagem. Rev Latinoam Enfermagem. 1995;3(1): 59-78.

4. Mark BA, Hagenmueller AC. Technological and environmental characteristics of intensive care units. Implications for job redesign. J Nurs Adm. 1994;24(4 Suppl):65-71.

5. The World Health Organization Quality of Life assessment (WHOQOL): position paper from the World Health Organization. Soc Sci Med. 1995;41(10):1403-9.

6. Universidade Federal do Rio Grande do Sul. Departamento de Psiquiatria e Medicina Legal. Divisão de Saúde Mental. Grupo WHOQOL. Versão em português dos instrumentos de avaliação de qualidade de vida (WHOQOL) 1998 [texto na Internet]. Porto Alegre: Universidade Federal do Rio Grande do Sul. [citado 2005 Ago 7]. Disponível em: http:/ /www.ufrgs.br/psiq/whoqol1.html

7. Fleck MPA, Louzada S, Xavier M, Chachamovich E, Vieira G, Santos L, Pinzon V. Aplicação da versão em português do instrumento abreviado de avaliação da qualidade de vida “WHOQOL -bref”. Rev Saúde Pública = J Public Health. 2000; 34(2):178-83.

8. World Health Organization . Programme on Mental Health. WHOQOL-BREF. Introduction, administration, scoring and generic version of the assessment. Field trial version. December 1996 [texto on the Internet]. [cited 2000 Oct 15]. Available from: http://www.who.int/mental_health/ media/en/76.pdf

9. BMJ. eBMJ-Statistics at Square One: correlation and regression. [texto on the Internet]. [cited 2007 Jun 01]. Available from: http://www.bmj.com/collections/ statsbk/11.dtl

10. Padilha MICS, Souza LNA. Qualidade de vida: reflexão de enfermeiras. Texto \& Contexto Enfermagem. 1999; 8(3): 11-26.

11. Silva VEF, Massarollo MCKB. A qualidade de vida e a saúde do trabalhador de enfermagem. Mundo Saúde (1995). 1998;22(5): 283-6.

12. Costa ES, Morita I, Martinez MAR. Percepção dos efeitos do trabalho em turnos sobre a saúde e a vida social em funcionários da enfermagem em um hospital universitário do Estado de São Paulo. Cad Saúde Pública. 2000;16(2):553-5.

13. Tepas DI, Barnes-Farrell JL, Bobko N, Fisher FM, IskraGolec I, Kaliterna L. The impact of night work on subjective reports of well-being: an exploratory study of health care workers from five nations. Rev Saúde Pública $=\mathrm{J}$ Public Health. 2004;38(Supl):26-31.

14. Noriega M, Gutiérrez G, Méndez I, Pulido M. Las trabajadoras de la salud: vida, trabajo y trastornos mentales. Cad Saúde Pública. 2004;20(5):1361-72.

15. Haddad MCL. Qualidade de vida dos profissionais de enfermagem. Rev Espaço Saúde. 2000; (2):75-88.

16. Matos E. Refletindo sobre a qualidade de vida no trabalho da enfermagem no Hospital Universitário. Texto \& Contexto Enfermagem. 1999;8(3):27-43.

17. Schmidt DRC, Dantas RAS. Qualidade de vida no trabalho de profissionais de enfermagem, atuantes em unidades do bloco cirúrugico, sob a ótica da satisfação. Rev Latinoam Enfermagem. 2006;14(1):54-60.

18. Pizzoli LML. Qualidade de vida no trabalho: um estudo de caso das enfermeiras do Hospital Heliópolis. Ciênc Saúde Coletiva. 2005; 10(4):1055-62.

19. Franco GP, Barros ALBL, Nogueira-Martins LA. Qualidade de vida e sintomas depressivos em residentes de enfermagem. Rev Latinoam Enfermagem. 2005;13(2):139-44.

20. Rocha SSL, Felli VEA. Qualidade de vida no trabalho docente em enfermagem. Rev Latinoam Enfermagem. 2004;12(1):28-35.

21. Oler FG, Jesus AF, Barboza DB, Domingos NAM. Qualidade de vida da equipe de enfermagem do centro cirúrgico. Arq Ciênc Saúde. 2005;12(2):102-7.

22. de Oliveira Filho GR, Sturm EJ, Sartorato AE. Compliance with common program Requirements in Brazil: its effects on resident's perceptions about quality of life and the educational environment. Acad Med. 2005;80(1):98-102.

23. Naumann VJ, Byrne GJ. WHOQOL-BREF as measure of quality of life in older patients with depression. Int Psychogeriatr. 2004; 16(2):159-73.

24. Horng YS, Hwang YH, Wu HC, Liang HW, Mhe YJ, Twu FC, Wang JD. Predicting health-related quality of life in patients with low back pain. Spine. 2005;30(5):551-5.

25. Becchi A, Rucci P, Placentino A, Neri G, de Girolamo G. Quality of life in patients with schizophrenia - comparison of self-report and proxy assessments. Soc Psychiatry Psychiatr Epidemiol. 2004; 39(5):397-401.

26. Hsiung PC, Fang CT, Chang YY, Chen MY, Wang JD. Comparison of WHOQOL-bREF and SF-36 in patients with HIV infection. Qual Life Res. 2005;14(1):141-50.

27. Costa G. Saúde e trabalho em turnos e noturnos. In: Fischer FM, Moreno CRC, Rotenberg L. Trabalho em turnos e noturno na sociedade 24 horas. São Paulo: Atheneu; 2003. p.79-98.

28. Benatti MCC, Nishide VM. Elaboração e implantação do mapa de riscos ambientais para prevenção de acidentes do trabalho em uma unidade de terapia intensiva de um hospital universitário. Rev Latinoam Enfermagem. 2000;8(5):13-20. 